\title{
Part 8: Service Delivery and Outcome Research
}

\author{
Barriers to Assistive Technology in Europe \\ Maurice Grinberg ${ }^{\mathrm{a}, \mathrm{b}, *}$ and Evgeniya Hristova ${ }^{\mathrm{a}, \mathrm{b}}$ \\ ${ }^{a}$ Department for Cognitive Science and Psychology, \\ New Bulgarian University, Montevideo str. 21, 1618 \\ Sofia, Bulgaria \\ ${ }^{\mathrm{b}}$ ASSIST - Assistive Technologies, Razvigor str. 3A, \\ 1421 Sofia, Bulgaria
}

Background: The results of the project "Barriers to the wider deployment of person centred technology in services for persons with disabilities," commissioned by the European Association of the Service Providers for People with Disabilities (EASPD) will be presented. The goal of the project was to identify what are the main barriers and consequently the potential facilitating factors that would enable the uptake of Person Centred Technology (PCT) in the social sector.

Method: The research work had two related parts a theoretical analysis of the barriers to PCT based on existing studies; and the design and deployment of a questionnaire aimed at gathering data from professionals in service providers for People with Disabilities (PwD) as to their perception of the identified barriers. The theoretical part is based on some of the most important recent reviews and authoritative sources of information on the topic. Fifteen groups of barriers were identified. The questionnaire was developed following the theoretical analysis and it covers all identified groups of barriers. The questionnaire was administered in 5 languages and was filled in by 137 professionals working in social service providers for people with disabilities from 18 European countries. 100 participants were included in the subsequent analysis.

Key results: The results of the questionnaire show that professionals in Europe are unanimous about the usefulness of PCT for PwD (mean rating of 6.4 out of 7). However, the data about the percentage of service providers' staff members using PCT is alarmingly low - $36 \%$ overall (about $25 \%$ for Central, Eastern, and
Southern Europe; $78 \%$ for Northern Europe). A similar large discrepancy between the North and the rest of Europe is also seen in the ratings about the good use of PCT in social service providers -5.8 (out of 7) for the North and 4.5 for Central, 3.4 for Eastern, and 3.6 for Southern Europe. However, the average rating for Europe (4.2) is not satisfactory.

Another big issue is that only $22 \%$ of the service providers' staff is rated as being expert in PCT $(50 \%$ for Northern Europe and about $12 \%$ for the rest of Europe). Additionally, according to the data, professionals working in SPPD have had on average less than 1 course in PCT during their formal education.

The analysis of the results shows that the most important barriers to the usage of PCT identified by the study belong to the following groups: Financial (except for Northern Europe with rating 4.5), Training, and Qualified Professionals barriers. Next in severity come the Resource, PwD and their Families' Knowledge and Skill, Environmental, and Legislative and Regulatory groups of barriers.

Conclusion: The results of the study show that despite some differences in perceived severity, the barriers to PCT in SPPD are common to Central, Eastern, Northern, and Southern Europe. The groups of barriers related to training and qualification with respect to PCT seem the most prominent for all Europe.

Facilitating measures need to be systemic, starting from the establishment of university curricula which will account for the exponential growth of PCT.

Keywords: barriers to assistive technology, professionals working with people with disabilities, training and qualification in assistive technology.

*Corresponding author. E-mail: mgrinberg@nbu.bg

Demanding Assistive Technology for Study and Work in Finland 2007-2018

Anna-Liisa Salminen $^{\mathrm{a}, *}$ and Jenna Mäkinen ${ }^{\mathrm{b}}$ 
${ }^{a}$ Research Department, The Social Insurance Institution of Finland, Nordenskiöldinkatu 12, 00250, Helsinki, Finland

Background: Finnish legislation guarantees disabled people the right to assistive technology (AT) that improves their ability to manage their daily activities independently. Responsibility for providing and financing such equipment falls upon the municipalities. However, if an illness or impairment makes working or studying impossible without personal AT, the Social Insurance Institution of Finland (Kela) can acquire the needed devices. Kela provides only particularly demanding and expensive devices. This includes equipment such as video magnifiers, Braille and largeformat displays and computers. Kela provides AT for people aged 16 or over. The aim of this study is to describe changes in the use of AT benefits paid by Kela in 2007-2018 in terms of different diagnostic and age groups and the cost of AT. In addition, the study describes reasons for rejecting AT applications.

Method: The study is based on Kela registers from 2007 to 2018 and a focus group interview for the five specialists responsible for the AT benefits at Kela.

Key results: The yearly number of disabled people who received $\mathrm{AT}$ acquired by Kela ranged between 550 and 600 persons in 2007-2018. Slightly over half of them were women. Most recipients belonged to the age group 16-19. However, the proportion of this age group in relation to the other age groups decreased yearly. Until 2012, most recipients were students, after which the majority were employed. The most common reasons for receiving the devices were related to vision loss and hearing loss. The number of devices acquired based on vision loss varied during the study period, whereas the number of devices acquired based on hearing loss increased. Other common conditions were neural diseases, such as cerebral palsy, but the number of acquired devices decreased over time. The total cost of the Kela-funded devices varied between 1.2 and 1.6 million euros, and individual AT solutionscost 2,000-2,500 euros on average. Kela rejected approximately one third [216-279] of the AT applications annually. The most common reasons for rejection were: the applicant did not have a sufficient disability, the device was not necessary for work or study, or the device was not demanding and expensive and needed to be financed by the applicant's municipality.

Conclusion: The yearly need for demanding and expensive AT acquired by Kela remains roughly the same. The number of recipients who are young or who study decreases over time. This may be explained by the fact that computer technology is now everyday technology and it is provided by schools or municipalities or bought by the persons themselves.

Keywords: Assistive technology, Provision, Study and Work.

*Corresponding author. E-mail: anna-liisa.salminen@ kela.fi

Augmentative and Alternative Communication Systems for Multilingual Contexts: A South African Perspective

Kerstin M. Tönsinga, ${ }^{a, *}$, Shakila Dada ${ }^{\mathrm{a}}$, Karin van Niekerk $^{\mathrm{a}}$, Georg I. Schlünz ${ }^{\mathrm{b}}$, Ilana Wilken ${ }^{\mathrm{b}}$, Jocelyn Mngomezulu $^{\mathrm{a}}$, Danél Hattingh ${ }^{\mathrm{a}}$, Ngwanamashiane R. B. Mothapo ${ }^{\mathrm{a}}$, Refilwe E. Morwane ${ }^{\mathrm{a}}$ and Nomadlozi B. Bokaba $^{\mathrm{C}}$

${ }^{a}$ Centre for Augmentative and Alternative Communication, University of Pretoria, Communication Pathology Building, Lynnwood Rd, Hatfield, Pretoria, 0002, South Africa

${ }^{\mathrm{b}}$ Human Language Technology Research Group, Meraka Institute, Council for Scientific and Industrial Research, Scientia 627-Jr, Meiring Naude Rd, Pretoria, 0081, South Africa

${ }^{\mathrm{c}}$ Department of African Languages, University of Pretoria, Humanities Building, Lynnwood Rd, Hatfield, Pretoria, 0002, South Africa

Background: In the field of augmentative and alternative communication (AAC) there is a dearth of knowledge to guide the design and implementation of AAC systems that give access to multiple spoken languages. With 11 official languages, South Africa is a context of linguistic and cultural diversity. However, due to a variety of socio-political and historical factors, AAC intervention and AAC technology are provided almost exclusively in English. Studies were therefore undertaken with the overall aim of developing augmentative and alternative communication (AAC) systems that would give access to expression in various South African languages. The aims of these studies were:

- To describe the perceptions and needs of stakeholders (rehabilitation professionals and persons using AAC) regarding non-English and multilingual AAC systems;

- To identify core vocabularies in three South African languages to guide vocabulary selection for nonEnglish and multilingual AAC systems. 
Method: The first study used a qualitative design. Online and face-to face focus groups were held with 15 purposively selected South African rehabilitation professionals working in the field of AAC to understand their perceptions of multilingual issues in AAC service provision including AAC systems and technology. Focus group data were analyzed using inductive thematic analysis.

In the second study, a predominantly quantitative survey design was used to obtain the perspectives of 27 multilingual South African adults using AAC on multilingual AAC issues. Participants were recruited through an empowerment program for adults using AAC and also through an email list. Data were analyzed using predominantly descriptive statistics.

The three core vocabulary studies made use of quantitative descriptive observational designs. Speech samples were collected from six isiZulu-speaking, six Sepedi-speaking and 12 Afrikaans-speaking preschoolers via body-worn audio recorders during routine preschool activities. Participants were selected using convenience sampling. The speech samples were transcribed and the linguistic units (words or morphemes, as dictated by the linguistic structure of the language) that were used with a frequency of at least $0.5 \%$ and by at least half of the participants were identified as the core vocabulary.

Key results: Both stakeholder studies indicated a need for AAC systems that allowed access to South African languages and also access to more than one language. Desired technology features included text-to-speech capabilities, text prediction and pre-stored vocabulary in appropriate South African languages, and the ability to code-switch seamlessly. Core vocabulary lists were identified comprising of linguistic units (words and/or morphemes) used most frequently and commonly by preschoolers, ranging from 239 words in Afrikaans, 226 words in Sepedi, to 213 morphemes or formatives in isiZulu. The influence of linguistic structure on AAC system design was also highlighted.

Conclusion: The results highlight the need for and desired features of AAC systems that give access to nonEnglish South African languages and also multilingual systems. Core vocabulary lists in the three languages can be used to assist with selecting vocabulary that allows for sentence construction and a level of linguistic generativity. These studies also highlight languagespecific and language independent design considerations for AAC systems giving access to multiple languages.
Keywords: Augmentative and alternative communication, Core vocabulary, Multilingual, South Africa, Stakeholder, System design.

${ }^{*}$ Corresponding author. E-mail: kerstin.tonsing@up. ac.za

\section{Access to Appropriate Assistive Technology in Less- resourced Settings: Argentina's Case \\ Author Silvana Contepomi \\ Argentine Assistive Technology Association}

Background: Assistive Technology (AT) promotes inclusion in people with disabilities across their lifespan. Although AT products are essential, successful access depends on integrated efforts related to end users ('people'), provision, personnel, and policy, considered the 5 'P's by the (WHO- GATE). Argentina, is an emerging-frontier country, Currently, $32 \%$ of its population live in poverty and disability is prevalent in $10.2 \%$ of the population. Most remote and vulnerable places lack appropriate processes to guarantee the rights of people with disabilities. The existing way of wheelchair distribution is massive and disconnected from the essential Service. Challenges are lack of: person-center approach - policy implementation services related to appropriate AT provision- maintenance of products. The aim of the pilot project research is to figure out if we can improve the results in inclusion with wheelchair provision service as a process in accordance to the WHO's paradigm.

Method: A Pilot project of AT provision was setup in Chaco Salteño, a vulnerable region of Argentina. A team of 3 experts composed by 1 physical therapist and 2 social workers defined the 7 phases for an adapted program on AT provision to ensure insertion of the WHO paradigm on the 5 "p" and the WHO's 8 steps of wheelchair provision. 7 Phases we planned in order to achieve our goals: 1: Advocating Policy/Provision, involve local government. 2: Personnel, communitybased rehabilitation training. 3: People, referral people in need of AT. 4: Personnel, training 2 local leaders in product preparation. 5: Provision, assessment and selection by expert. 6: Product, funding, donations were accepted. 7: Provision/Personnel, follow up every 3/6 months. Interview to obtain results.

\section{Key results:}

- Coordinated efforts with the government to advance 5 "P" understanding the population's needs

- 32 users were provided with appropriate wheelchair and training 
- 140 health agents trained in CBR increasing awareness shown in number of cases

- 2 local leaders instructed in product maintenance. Diminish abandon products

- Increased community participation / inclusion(fig.1)

Conclusion: This Program is just a pathway ensuring appropriate AT products and service provision for the vulnerable community. The benefits of AT should be known by the stakeholders involved with disabled people throughout their lifespan. Future perspectives:

- Monitoring and evaluation programs in order to build the evidence of the cost and benefits of AT.

- Building capacity goes hand in hand with the integration of these services into existing healthcare and social programs for ensuring equitable access to appropriate AT

- Program can be replicate in less resource settings in Latin America.

Keywords: Less-resourced settings, Service steps, wheelchair provision

*Corresponding author. E-mail: Silvana@asistiva. com.ar

Availability and Awareness of Assistive Products in Bangladesh from the Perspective of Rehabilitation Professionals

Mehedi Hasan Khan ${ }^{\mathrm{a}, *}$, Hidehisa $\mathrm{Oku}^{\mathrm{a}}$ and Mitsuhiko Sano $^{\mathrm{a}}$

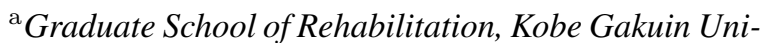
versity, 518 Arise, Ikawadani-cho, Nishi-ku, Kobe, 651-2180, Japan

Background: Bangladesh is considered as lowermiddle income country with approximately 164 million population. Although $9.1 \%$ of total population are experiencing disability, the production and distribution of assistive products (AP) have not been sufficient so far. Offering APs in the market and making appropriate choice to effective use of APs are two burning problems of Bangladesh. Affordable domestic production and imports from overseas may minimize demand problem but adequate knowledge of rehabilitation professionals (RP) about APs is indispensable for maximizing services. To improve this situation, this research was carried out first to reveal the kind of available APs in Bangladesh along with measuring the level of awareness of RPs about APs.

Method: As there is no any business enterprise to produce APs in Bangladesh, Center for the Rehabilitation of the Paralysed (CRP), the only rehabilitation center and Center for the Development of the Disabled (CDD), the prominent disability related organization play vital role in production and distribution of APs. On the other hand, WHO has developed a 50 Priority Assistive Products List (APL) to improve access to high quality, affordable APs for all in 2016. In this research, that APL was selected as a list of standard APs. The kind of available APs in Bangladesh, has been surveyed by analyzing statistical data published by CRP and CDD. To measure the level of awareness of RPs about APs, all the RPs of CRP were surveyed by questionnaire. The total participants were 115 RPs which included PT-52, OT-32, SLT-16, and P\&O-15. In the questionnaire participants were asked about their awareness of 50 APs from APL along with their professional information. This research has been carried out from September 2018 to April 2019 with the permission of CRP Research Ethics Review Committee.

Key results: The survey result revealed that, 23 out of 50 kinds of APs from the APL were available in Bangladesh and all those available APs were from Mobility, Communication and Environment categories of APL. In terms of awareness of RPs about APs, only 1 participant from $\mathrm{P} \& \mathrm{O}$ knew all 50 APs from APL properly. However, there were 7 more participants (OT3, SLT-3 and P\&O-1) who knew 40+ APs from APL. The study also indicated that only 9 participants knew all the 23 APs available in Bangladesh. Statistical analysis indicated that the awareness about APs in OT was significantly higher than that in PT. The survey further denoted that $90.5 \%$ of RPs didn't have any knowledge about database of APs although 48.0\% RPs obtained information of latest APs from internet. The study also found that $81.0 \%$ RPs never had an opportunity to participate any AP related seminar/conference outside Bangladesh and $13.1 \%$ RPs didn't have any scope to get information about latest APs.

Conclusion: The preliminary result of our research indicated that, the numbers of available APs in Bangladesh were fewer than the APL and the awareness level of RPs about APs was inadequate. Establishment of worthy environment to learn more APs effectively is highly needed along with solving the priority issues.

Keywords: Rehabilitation professional, Bangladesh, Assistive Products List.

${ }^{*}$ Corresponding author. E-mail: mkhansm02@gmail. com 
Assistive Technology Access: A global concern in the Canadian context - stakeholder perspectives on unmet needs, gaps in services, and ethical, social, and policy issues

Natalia Zdaniuk*, Rosalie Wang, Evelyne Durocher and Michael Wilson

Department of Occupational Science \& Occupational Therapy, University of Toronto, 160-500 University Ave., Toronto, ON, M5G 1V7, Canada

Background: As technology development advances, access to assistive technology (AT) remains a global concern. The World Health Organization has determined an urgent need for AT policies that enable equitable access nationally and regionally. In Canada, there are unmet needs and access to AT remains challenging, raising equity concerns. Programs offering AT are fragmented and complex, resulting in barriers for consumers and healthcare providers. There is a clear urgency for coordinated research and policy implementation for AT access. This study is part of a larger project to understand and advance Canadian policies for AT access. This study covers a previous gap in research by engaging stakeholder perspectives about AT in Canada in relation to policy, provision, and services. The purpose of the study is 1) to examine how AT is accessed by Canadians and identify funding/service gaps, and 2) to explore the perceived ethical, social, and policy issues affecting AT access and adoption. The findings will contribute to better aligning policies/services with the perceived social and ethical values of Canadians and improve equitable access through policy implementation.

Method: In this study we undertook 30 key informant interviews with Canadian policymakers and stakeholders representing federal, provincial, and territorial jurisdictions, and organizations operating nationally. Using a purposive expert sampling framework, the researchers aimed for 2-3 representative policymakers/stakeholders from 14 Canadian jurisdictions. Using thematic analysis, the researchers reviewed and coded interview transcripts, refining themes based on ongoing review and discussion. Themes were sorted based on value placed by respondents, frequency of appearance, and significance to the research purpose.

Key results: Three prominent needs emerged from the interviews, raising key issues to address in future policy proposals: 1) The need for a more holistic approach, offering a national-scale intervention to currently silo-ed services that limit AT access and do not reflect a client's full range of needs; 2) The need for client-centred policies and programs, offering broader inclusion and prioritizing choice and independence; 3) The need for capacity building, including education/awareness campaigns, and training for practitioners, clients, and caregivers - including those facing barriers to information, such as rural populations and newcomers to Canada. Under these themes, multiple gaps in service/provision/policy were identified, and a list of groups with unmet needs was developed. Respondents also identified proposals to address these concerns, including building national standards, providing subsidies, and developing centres of excellence for education.

Conclusion: The findings show what stakeholders and policymakers perceive as the greatest concerns for access to AT in Canada, highlighting the barriers and gaps in accessibility, as well as offering strategies for policy implementation and service improvement. The study offers practical implications to guide ongoing research engagement with policymakers and stakeholders. While based in a Canadian context, the study addresses a global concern and offers a meaningful contribution to the AT field by engaging in a process to improve access to AT. This work contributes to broader social change by addressing the call for accessible ATs to operate as mediators of sustainable and equitable development internationally.

Keywords: Policy, Access, Equity, Client-centred, Capacity-building

Continuous Outcome Scaling: A Discriminative Method for Person-Centered Assistive Technology Outcomes Studies

Natasha Layton*, Karen Rispin ${ }^{\mathrm{b}}$ and Vicki Sheafer ${ }^{\mathrm{c}}$

${ }^{a}$ Department of Health Professions, Swinburne University of Technology, John St, Hawthorn, Vic 3122, Australia

${ }^{\mathrm{b}}$ Department of Biology, LeTourneau University, 2100 S. Mobberly Avenue, Longview, TX, USA

${ }^{\mathrm{c}}$ School of Psychology \& Counseling, LeTourneau University, 2100 S. Mobberly Avenue, Longview, TX, USA

Background: Accurate measurement is a critical element of assistive technology (AT) provision. Outcome measures which are useful in both clinical settings and field studies must have evidence which confirms their reliability and validity. This paper considers the appropriateness of using a graded visual analogue scale with grades as anchors for the scale. Specifically, the abil- 
ity of graded visual analogue scales to discern meaningful differences for a population of young manual wheelchair users and their practitioners within lowand middle-income countries.

Method: This paper reviews the literature on continuous scaling applicable to assistive technology outcomes. We then describe the development of graded visual analogue scales using grades as anchors within a mixed methods protocol. Studies drawing on eleven datasets related to wheelchair and prosthetic/orthotic functioning are reviewed to identify important parameters for validation and to critically evaluate tool performance.

Key results: Graded visual analogue scales with grades are anchors demonstrate strong ability to discern meaningful difference. This method provides data with enough detail to make it useful usable for clinical and design responses.

Conclusion: Meaningful outcomes measurement with useable tools is critical to evaluate and build assistive technology access globally, and a high priority considering the UN Sustainable Development Goals. This paper proposes a protocol for use of graded visual analogue scales and demonstrates evidence of its reliability and validity across a range of studies. The graded visual analogue scale approach to outcome measurement offers relevant, rapid and sensitive measures, widely applicable. The paper concludes with considerations for the potential use of continuous scaling from the twin perspectives of consumer usability and utility in low- and middle-income countries.

Keywords: outcomes, disabilities, person-centered, continuous

${ }^{*}$ Corresponding author. natasha@ natashalayton.com. $\mathrm{au}$

Towards a Successful Match Between User and Assistive Technology: A Correlational Study on User's Satisfaction, Perceived Effectiveness, and Psychosocial Impact of an Assistive Solution

Stefano Federici ${ }^{\mathrm{a}, *}$, Eugenio Valenzano ${ }^{\mathrm{a}}$, Maria Laura Mele $^{\mathrm{a}, \mathrm{b}}$ and Fabrizio Corradi ${ }^{\mathrm{c}}$

${ }^{a}$ Department of Philosophy, Social \& Human Sciences and Education, University of Perugia, Perugia, Italy

${ }^{\mathrm{b}}$ Myèsis, Research and Development Company, Rome, Italy

${ }^{\mathrm{c}}$ Leonarda Vaccari Institute for Rehabilitation Integration and Inclusion of Persons with Disabilities, Rome, Italy
Background: This work is an exploratory study to investigate the relation among three main psychosocial factors measured after an Assistive Technology (AT) system provision: (i) the user's satisfaction, (ii) the perceived effectiveness, and (iii) the psychosocial impact of the assistive solution. The main objective is to understand whether the correlated psychosocial measures can predict a successful outcome of the AT assignation process. The study embraces the ICF's biopsychosocial perspective of disability and the Matching Persons and Technology model by adopting the Assistive Technology Assessment (ATA) process, an ideal model for the effective outcome of AT assessing and provision (Federici \& Scherer, 2018).

Method: The study was conducted in an AT service delivery center by following the guidelines provided by the ATA model. Fifty-five subjects $(72.7 \%$ males, min 4 - max 14 years old) participated in the study, which consisted of five main phases. Two preliminary phases focused on the analysis of participants needs with the aim to identify the best AT solution through a psychological counseling process and the administration of the Matching Assistive Technology \& Child (MATCH; Federici et al., 2009). Then, the third phase of postassignation focused on the outcome of the assistive solution assignation process measuring the user's satisfaction and the perceived effectiveness, as well as the functional independence, well-being, and quality of life. The factors were assessed through (i) the Quebec User Evaluation of Satisfaction with assistive Technology (QUEST; Demers et al., 2002), (ii) the Individually Prioritized Problem Assessment (IPPA; Wessels et al., 2002), and (iii) the Psychosocial Impact of Assistive Devices Scale (PIADS; Jutai and Day, 2002). A final post-assessment phase consisted in providing the users with tailored training for the use of the assigned AT and in conducting interviews to caregivers (teachers and educators) and peers to investigate the impact of the AT solution in the users' milieu.

Key results: Findings after the AT system provision showed high levels of user's satisfaction (Total QUEST $M=4.80$; QUEST device $M=4.71$; QUEST services $M=4.99$ ), an increase in quality of life levels and a decrease of about $40 \%$ of the perceived difficulty of use. A highly significant positive correlation among the three questionnaires QUEST, IPPA, and PIADS was found $(0.473 \leqslant r \leqslant 0.762 ; p \leqslant 0.01)$. A significant linear relationship between the questionnaire scores and the abandonment rate shows that questionnaire scores can predict each other $\left(0.224 \leqslant R^{2} \leqslant\right.$ $0.581 ; p \leqslant 0.01)$. Finally, the AT assessment outcome showed a $5.17 \%$ rate of abandonment. 
Conclusion: A positive relation among psychosocial factors of users' satisfaction, perceived effectiveness, and the psychosocial impact of the assistive solution highlighted a statistical dependence among the three different metrics recommended by the ATA model for the post-assignation assessment process (QUEST, IPPA, and PIADS), also explaining the strength of each psychosocial factor on the AT assessment outcome in terms of abandonment of the assistive device. Future works will extend the age range and focus on designing a mathematical model for predicting the percent probability of AT getting abandoned by the user, immediately after the post-assignation assessment process.

Keywords: Assistive Technology Assessment model, Matching Assistive Technology \& Child - MATCH, QUEST, IPPA.

*Corresponding author. E-mail: stefano.federici@ unipg.it

What is the pay-off? Usability and cost benefit of assistive technology at workplace

Sajay Arthanat and Alyson Messina

Occupational Therapy, University of New Hampshire, USA

Background: Assistive Technology (AT) devices enable individuals with disabilities (IWDs) to fulfill work roles and engage in meaningful employment. However, the employment rate of IWDs in the United States is significantly lower $(18.7 \%)$ compared to that of ablebodied individuals $(65 \%)$. A major factor here pertains to barriers with implementation of AT devices. To highlight the role of AT, we conducted a comprehensive survey of employees with disabilities on their use and utilization of workplace AT. Specifically, the study sought to: a) identify AT devices that are being used at work; b) examine how well the devices effectively and efficiently fulfilled work roles; c) examine perceived skills and accommodations for use of AT; and above all; d) analyze the cost-benefit of integrating AT devices at workplace.

Method used: The survey was developed using a pilottested work-driven framework, the Usability Scale for Assistive Technology-Workplace (USAT-W). The framework first delineates the work roles of the employee, focuses on tasks within each role, and then considers the AT support needed to fulfill each role. The effectiveness and efficiency with which the employee interacts with the AT devices, as well as the skills involved and accommodations received for each device within the roles, are then evaluated. This framework was adapted into the survey, pilot tested with a small cohort of employees with disabilities, and then uploaded on Qualtrics survey database. We included an additional section seeking information on the employee's income and total cost with acquisition, training, and service for each AT device. Convenience sampling was used to recruit participants across the United States from multiple sources- Qualtrics health panels, disability support networks, and Centers for Independent Living.

Key Results: 206 employees with disabilities who used AT (with an average 8.1 years of work experience) completed the survey. The three main categories of AT were worksite modifications (25.7\%), devices for mobility $(23.8 \%)$ and computer access devices $(21.8 \%)$. Nearly $90 \%$ of respondents rated themselves to be "productive" to "very productive" in all work roles involving AT. About $84 \%$ to $93 \%$ stated their AT devices to be "effective" or "very effective", and "efficient" or "very efficient" for fulfilling their work roles. Significant correlations (r ranging from 0.3 to $0.6 ; p<0.01$ ) were detected between AT skills, accommodations, and work productivity. In contrast to the average annual income of $\$ 47,135$ (Std.dev $=$ $\$ 33,558)$, the average total cost of AT devices was $\$ 7074$ (Std.dev $=\$ 15,868$ ), almost a 7:1 ratio. Despite the high variance, the one-time total cost of AT devices was a much smaller fraction of the respondents' annual income across all impairments and job categories.

Conclusion: These findings indicate the breadth and role of AT devices at work. The economic benefits of providing AT with respect to income and earning potential far surpass any associated implementation costs. While past research has delineated barriers to AT provision, underscoring the value and economic pay-off of workplace AT is critical to highlight the role of AT to service providers, employers, and policy makers. ${ }^{*}$ Corresponding author. E-mail: sajay.arthanat@unh. edu

Technology's Impact on Tasks of Employees with Disabilities in Germany (2006-2017)

Sabrina Inez Weller

BIBB Federal Institute for Vocational Education and Training, Robert Schuman Platz 3, 53715 Bonn, Germany

Background: The ongoing computerization of our economy leads to a transformation of the world of 
work. New technologies and new working requirements result in changes in employees' task composition. According to the Routinization hypothesis, the increasing use of computer technologies in the workplace is leading to a complementary of non-routine (analytic and interactive) tasks and substitution of routine (manual and cognitive) tasks). This paper analyses the influence of the computerization (the use of computer as main work tool) on the tasks of employees with disabilities. It is examined whether the technological change is leading to the same shift towards more non-routine tasks and to the same substitution and complementary effects of technology in workplace among employees with disabilities as it is among those without impairments between 2006 and 2017.

Method: We use representative data form the German BIBB/BAuA Employment Surveys 2006 and 2017, where 20,000 gainfully employed people were asked about their Qualification and Working Conditions. We run separate regression models for employees with and without a certified disability in the years 2006 and 2017, where we analyse the influence of computer usage (independent variables) on different task categories (routine, manual, interactive and analytical tasks; dependent variables).

Key results: Our results show no significant differences in the technology usage of employees with and without disabilities on workplace. Moreover, the impact of technologies on the tasks carried out do not differ significantly between disabled and non-disabled employees.

Conclusion: The analyses confirm the Routinization hypotheses for disabled employees: the usage of technology on workplace is leading to the same shift towards more non-routine tasks and to the same substitution and complementary effects on tasks for disabled and non-disabled employees. One explanation for the fact that computerization affects in the same way people with and without disabilities could be that the data used focusses on persons who are integrated in the general labour market. In future research we will analyse the effect of various types of technology (e.g. tools for communication and information management, input and output devices for computers) on the tasks of employees with different impairments to find more differentiated results.

Keywords: Disability, Labour market participation, Routinization.

*Corresponding author. E-mail: weller@bibb.de
Assistive Technology Outcome Measures: A Review of Recent Literature

Claudia Salatino ${ }^{\mathrm{a}, *}$, Renzo Andrich $^{\mathrm{a}}$ and Lorenzo Desideri $^{\mathrm{b}}$

a IRCCS Fondazione Don Carlo Gnocchi, Milan, Italy

bAIAS Bologna Onlus, Piazza della Pace 4/A, 40134 Bologna, Italy

Background: Effects, costs and economic impact of assistive technology are indicated among the top priorities in the Global Priority Research Agenda on Assistive Technology, published by the World Health Organization in 2017. Internationally validated AT outcome measures can be useful to measure whether AT interventions lead to successful outcomes at individual level, and more in general to collect evidence about AT appropriateness and cost-effectiveness. The authors who work in AT assessment teams of rehabilitation and AT centers - were interested in finding out outcome measures applicable to any AT categories, which could be used in the follow-up stage of AT interventions in daily practice.

Method: A literature overview was undertaken in order to identify validated outcome measurement tools, in use among the AT communities, that could be applied to any individual AT intervention. Three AT experts identified a list of 13 journals and 3 conference proceedings whose field of interest included AT, and analyzed articles published in their 1312 releases between 2003 and 2016. First, 459 articles were retrieved that contain the terms "OUTCOME" or "MEASURE" or "EFFECTS" or "TOOL". Second, each expert read the abstracts and assigned a relevance score. Papers were considered "relevant" if the abstract declared that the described instruments were intended to measure the outcome of individual AT interventions; otherwise they were scored as "not relevant". Third, the 53 articles scored as "relevant" by all the three experts were analyzed in depth and the data describing the study and the instruments were extracted and coded. For example, each instrument was classified according to whether it was: 1) intended for general use in rehabilitation programs, 2) designed to be applied to specific categories of assistive products, and 3) applicable to any AT products.

Key results: 86 instruments were reported in the 53 selected articles. However, only 5 instruments were found to be outcome measures actually applicable to a broad range of assistive products: PIADS, QUEST 2.0, FIATS, IPPA and SCAI. These instruments were described in 29 articles, which indicates they are 
quite widely known and used in several Countries. These instruments investigate different outcome domains - respectively - psychosocial impact, satisfaction, caregivers' burden, perceived effectiveness and social costs.

Conclusion: Outcome measures are extremely important to demonstrate the AT fundamental role and added value, to quantify their impact and to inform funding decisions. However, a very simple set of easy-toadminister measures should be chosen to make the measurement sustainable in clinical practice. The five instruments identified by this study seem to meet this need; they also investigate different outcome domains whose combination provides a comprehensive insight into the AT impact on the user's life. The authors are currently engaged in further research to seek the best compromise between making outcome measurement sustainable in clinical practice and collecting the whole spectrum of data that can fully enlighten the AT impact.

Keywords: assistive technology intervention; outcome measurement; follow-up

*Corresponding author. E-mail: csalatino@dongnocc hi.it

\section{Outcome of Provision of "Uncomplicated" Assistive Devices to Older People in the Faroe Islands - an IPPA pretest-posttest Study}

Turið Jacobsen, Maria Skaalum Petersen and Gro Gade Haanes

The University of the Faroe Islands, Faculty of Health Sciences

Background: Researching the outcomes of assistive devices (ADs) is important for clinical and policy decision-making. The Faroe Islands have an ageing population, and there is a lack of knowledge about outcome of ADs for older people. Older people recieve most of the ADs from the publicly funded ADcentre. This study aimed to evaluate to what extent older people experience diminished difficulty in performing daily activities due to provision of uncomplicated ADs, e.g bath chairs and rollators. Additionally, the aim was to examine possible association between effect and "sociodemographic factors" and "difficulty at baseline". This is the first study of AD provision in the Faroe Islands.

Method: The design was a pretest-posttest AD intervention study. It was conducted in five out of seven municipal districts and ran for eight months. Seven municipal OTs collected data in their districts. Inclusion criteria: citizens 67 years and older living at home who wished ADs to perform daily activities. Exclusion criteria: citizens without sufficient cognitive or hearing functioning necessary to participate in an interview and citizens with massive need for care. Data collection: The effect was evaluated by means of the Individually Prioritised Problem Assessment (IPPA). IPPA assesses to which extent activity problems identified by $\mathrm{AD}$ users are diminished after recieving ADs. At baseline, the participant identified and rated their activity problems regarding importance and difficulty and re-evaluated them at follow-up 3.5 months after receiving the ADs. The scores range is $1-25$ points. A high score demonstrates a large difficulty. The effect is the difference between mean baseline score and mean follow-up score. Max possible difference is 24 points, which demonstrates a large effect. Intervention: municipal OTs assisted with the application for the ADs from the state $\mathrm{AD}$-centre, that might address the activity problems. When the ADs were recieved, the OT paid one visit to test and adjust the ADs, and to instruct in using. The overall effect was assessed with a paired t-test. The effect size was calculated and interpreted according to Cohen's d: an effect size of 0.20 is small, 0.50 is moderate and 0.80 or more is large. One-way ANOVA was used to explore association between the effect and the determinants.

Key results: In total 52 persons, 37 women and 15 men aged 69-93, mean age 83 years participated in the study.

Mean baseline total score was $17.73(\mathrm{SD}=4.09)$, mean follow-up score was $9.14(\mathrm{SD}=3.41)$. Mean reduction in difficulty was 8.59 points (95\% CI: $7.37-$ 9.81) $(p \leqslant 0.001)$. At the individual level the effect was -5-20 points. Effect size was 1.96 . Only "high difficulty at baseline" was associated with large effect ( $p<$ 0.001).

Conclusion: The effect size by 1.96 was large and shows positive effect of the AD provision. Only difficulty at baseline was associated with large effect. The variation of effect highlights the importance of evaluating $\mathrm{AD}$ interventions on an individual level. Further studies are needed to investigate which factors in the service delivery proces influence the outcome. This could provide the authorities with evidence in the planned re-evaluation of the AD service.

Keywords: assistive devices, outcome, older people, daily activities.

${ }^{*}$ Corresponding author. E-mail: turidjac@ @otmail.com 
Developing an administration manual for the electronic Mobile shower commode ASsessment Tool (eMAST): A case study

Emma Friesen $^{\mathrm{a}, \mathrm{b}, *}$

${ }^{a}$ Raz Design Inc, 22 Howden Road, Toronto, Canada

bindependent Researcher, Amsterdam, The Netherlands

Background: In Assistive Technology (AT), there is an ongoing need to develop, implement, and report on outcomes of AT provision and service delivery. In the past decade, efforts have largely focused on developing and validating new outcomes measurement instruments for use in real-world service delivery settings. In order to utilize these in practice, experts in instrument development recommend that documentation, in the form of administration guides or user manuals, should also be produced. While many resources exist to support instrument development, comparatively few appear available for developing administration or user manuals. Such documents serve as a single, comprehensive repository of information on an instrument to facilitate their uptake and use in practice. The purpose of this paper is to describe the development of an administration manual to accompany a recently-developed outcomes measure, the electronic Mobile shower commode ASsessment Tool version 1.1 (eMAST 1.1). The eMAST, first published in 2016, assesses mobile shower commode usability from the perspective of adults with spinal cord injury. This paper serves as a practical case study on developing an administration and user manual for researchers undertaking such work.

Method: The author reviewed the following resources to identify necessary elements for an administration manual: published literature on scale development; published guidelines from online repositories and distribution platforms on administration manuals for instruments hosted on their sites; data extraction domains used in published reviews of AT outcomes measures; and published administration or user manuals for existing, validated outcomes measures for wheeled mobility and usability.

Key results: The following elements were identified as necessary for inclusion in the eMAST administration manual: administrative details of the instrument including its full name, authors and their contact details, copyright status, authorized distributors, requirements for training or accreditation of administrators prior to use, costs or fees for licensing and use, reporting and disclosure requirements, and recommen- dations for citations in publications; background information on the development and validation history of the instrument, including a summary of key development phases, populations used for validation, and potential limitations or threats to validity; psychometric properties as reported in published studies; administrative properties as reported in published studies; instructions for use and guidance on administering the instrument, including recommended delivery format/s and availability of templates or formatted instruments (i.e. electronic, online, paper), recommended timing for administration (e.g. immediately after using the AT or after a week of use), scoring of the instrument, and interpretation of sub- and total- scores. These elements were then used by the author to create a draft version of the eMAST Administration Manual. The draft will be subject to external review by potential administrators of the eMAST in both research and service delivery settings.

Conclusion: This case study describes development of an administration manual to accompany a validated outcomes measurement instrument. The comprehensive manual contains information on the instrument's background, psychometric and administrative properties, and administration procedures for the eMAST. The manual will next be subject to external review by experts.

Keywords: Outcome measures; Questionnaires; User administration manual; mobile shower commode chairs; Practitioner research.

${ }^{*}$ Corresponding author. E-mail: efriesen@razdesigni nc.com

\section{Cross-Walking the Matching Assistive Technology to Child Assessment to the ICF Model}

Susan A. Zapf ${ }^{\mathrm{a}, *}$ and Marcia J. Scherer ${ }^{\mathrm{b}}$

${ }^{a}$ Post-Professional Occupational Therapy Program, Rocky Mountain University of Health Professions, Provo, UT, USA

${ }^{\mathrm{b}}$ The Institute for Matching Person and Technology, Webster, NY, USA

Background: The Matching Assistive Technology to Child-Augmentative Communication Evaluations Simplified (MATCH-ACES) assessment incorporates the International Classification of Functioning, Disability and Health (ICF) model within the assessment framework in order to provide a common language and structure for assistive technology assessments. The purpose of this descriptive analysis is to cross-walk 
constructs of the MATCH-ACES assessment to the ICF in the areas of educational participation, academics, and functional living skills with a special emphasis on intellectual disabilities. The authors hypothesize that the MATCH-ACES constructs/questions will correlate with the ICF body \& structures codes, activities and participation codes, environmental codes, and predisposition factors that influence assistive technology use.

Method: The lead author created a preliminary crosswalk and electronic database and cross-referenced the MATCH-ACES assessment questions (constructs) to the ICF domains using a typology mapping table and established criterion rules. The crosswalk was examined by two additional reviewers to determine the level of agreement of the MATCH-ACES constructs mapped to the ICF domains. Feedback was incorporated into the second revision of the crosswalk and the primary author developed a concept map visualizing the crosswalk of both assessments with a focus on intellectual disability.

Key results: Preliminary results indicated a high percentage of agreement between both reviewers in cross walking the MATCH-ACES assessment to the ICF model. Specific outcomes of this analysis include the development of common AT language across universal assessments in healthcare and education and the focus on participation for children and youth with impairments that impede function. This is the initial stage in mapping both assessments to develop a standard AT assessment with universal language for healthcare providers. Further research is needed to conduct a larger analysis of the constructs mapped to establish further reliability of this process.

Conclusion: A preliminary analysis found the MATCH-ACES assessment process cross-walks with the ICF model in both the cognitive components of the needs analysis form and the predisposition scales. A second contribution relates to the need to develop a standard assessment process that uses evidence-based research and universal terminology.

Keywords: Matching Assistive Technology to Child (MATCH), International Classification of Functioning, Disability and Health (ICF), Crosswalk

*Corresponding author. E-mail: suezapf@me.com

Translation and Preliminary Validation of the Italian Version of the Family Impact of Assistive Technology Scale for Augmentative and Alternative Communication (FIATS-AAC.it)
Riccardo Magni ${ }^{\mathrm{a}, *}$, Elvira Veglio ${ }^{\mathrm{b}}$, Jessica Carloni $^{\mathrm{a}}$, Steve Ryan ${ }^{\mathrm{d}}$ and Lorenzo Desideri ${ }^{\mathrm{c}, *}$

${ }^{a}$ COAT Centro Orientamento Ausili Tecnologici Ass. Onlus - Piazza Garibaldi 506039 Trevi, Italy

${ }^{\mathrm{b}}$ AUSL Umbria 1, Via della Pallotta 42, Perugia, Italy ${ }^{\mathrm{c}}$ AIAS Bologna Onlus, Piazza della Pace 4/A, 40134 Bologna, Italy

${ }^{\mathrm{d}}$ Holland Bloorview Kids Rehabilitation Hospital, University of Toronto, Toronto, Canada

Background: Developing effective communication skills is crucial for social and cognitive development, everyday functioning and inclusion in society. Interventions to improve communication skills of children with communication-related disabilities make use of alternative and augmentative communication (AAC). Recent studies have shown the effectiveness of these interventions on a number of clinical conditions; less clear, however, is whether the benefits from AAC interventions extend also to children's families. Understanding the impact of AAC on the lives of children and their families is important because family members often play a fundamental role in the successful integration of an AAC system into child's life. The Family Impact of Assistive Technology Scale for Augmentative and Alternative Communication (FIATS-AAC) is an emerging, reliable parent-reported outcome measure designed to detect the functional impact of AAC interventions on family systems. The present contribution reports on development and evaluation of the measurement properties of an Italian version of the FIATSAAC (hereafter, FIATS-AAC.it).

Method: The authors translated and adapted the FIATS-AAC to the Italian context by following a standard linguistic validation protocol that employed a translation-back-translation technique. The initial FIATS-AAC.it was administered by either phone or face-to-face encounters to 30 parents or caregivers of children with chronic disabilities who were aged 3 to 18 years. Parents completed the FIATS-AAC.it twice with a one-week interval. During the second administration, parents also completed the Impact on Family Scale (IFS) to assess construct validity. The IFS is a published measure of the psychosocial consequences of having a child with a disability on the family.

Key results: Test-retest reliability for the FIATSAAC.it total scale was acceptable (intraclass correlation coefficient (ICC) $=0.98$ (absolute agreement). Internal consistency for the total scale was also acceptable (Cronbach's alpha $=0.87$ ). Individual alpha values for FIATS-AAC.it subscales ranged from 0.19 to 0.87 , with 9 of 13 dimensions exceeding the reco- 
mmended threshold for homogeneity (alpha $\geqslant 0.7$ ). As hypothesized, correlational analyses revealed a negative correlation between FIATS-AAC.it and IFS $(r=$ $-0.73 ; p<0.01)$, suggesting that the measure taps into a construct related to the psychological consequences of having a child with a disability. However, parents and AAC professionals reported the 89-item FIATSAAC.it to be lengthy (about 20 minutes to complete).

Conclusion: The FIATS-AAC.it is a promising Italian tool to assess the child and family functioning in areas that may be impacted by the introduction of AAC interventions. The present study provides support for the reliability and validity of the total scale. The measurement properties of the FIATS-AAC.it generally align with those reported for the original English version. Further research is needed to confirm these findings and other properties (responsiveness). In addition, a shorter version would be a welcomed alternative for both AAC professionals and parents.

Keywords: AAC, children with complex communication needs, outcome measurement, effectiveness, family-centered services.

*Corresponding author. E-mail: mail@coatnet.it, 1desideri@ausilioteca.org 